\title{
Knowledge, Attitude, and Practices Among Lebanese Obstetricians and Gynecologists With Respect to COVID-19 and Pregnancy
}

\author{
Dalal Youssef ${ }^{1,2}, \mathrm{BSc}, \mathrm{MSc}, \mathrm{MPH}, \mathrm{PhD}$
}

${ }^{1}$ Preventive Medicine Department, Beirut, Lebanon
${ }^{2}$ Institut de Sante Publique d'Epidemiologie et de Developement (ISPED), Universite de Bordeaux, Bordeaux, France

\section{Corresponding Author:}

Dalal Youssef, BSc, MSc, MPH, PhD

Preventive Medicine Department

Ministry of Public Health

Beirut

Lebanon

Phone: 96171239633

Email: esumohleb@gmail.com

\begin{abstract}
Background: The COVID-19 pandemic has seriously disrupted the daily life of the general population, particularly the life of pregnant women. Since obstetricians and gynecologists (OBGYNs) are often the primary health care providers during pregnancy, they play a critical role in preventing and managing COVID-19 in their patients.

Objective: This study aimed to assess the knowledge, attitudes, and practices of OBGYNs with respect to COVID-19 and to identify existing gaps that need to be addressed to improve patient and occupational safety.

Methods: A cross-sectional study using a web-based survey was conducted among Lebanese OBGYNs during the rapid growth of the COVID-19 pandemic in Lebanon between October 20 and November 20, 2020. The analysis was performed using the SPSS software. Knowledge, attitude, and practice scores were computed. A good level of knowledge was considered when $80 \%$ of answers from the respondents were correct.

Results: A total of 279 OBGYNs participated in the survey, of whom 57\% were men. The majority of OBGYNs (64.2\%) were more than 45 years of age and married $(79.9 \%)$ and had extensive work experience $(70.3 \%)$. Only $28.3 \%$ were reluctant to provide medical care for patients with COVID-19. Most of them were afraid of contracting COVID-19 or transmitting COVID-19 contracted through occupational exposure to their family members and $42.3 \%$ felt overwhelmed. Of the OBGYNs, $62.7 \%$ considered the policies implemented by the Ministry of Public Health to be sufficient. The majority of OBGYNs had a good level of knowledge in different basic and specific domains related to COVID-19 and pregnancy. Furthermore, the practice score was good in all relevant aspects (personal, clinic, and patient).
\end{abstract}

Conclusions: The high knowledge and practice scores among Lebanese OBGYNs indicate a strong commitment from these physicians to fulfill their responsibilities toward themselves and their patients during the COVID-19 pandemic.

(iproc 2022;8(1):e36533) doi: $\underline{10.2196 / 36533}$

\section{KEYWORDS}

knowledge; attitudes; practices; pregnancy; COVID-19; obstetricians; gynecologists

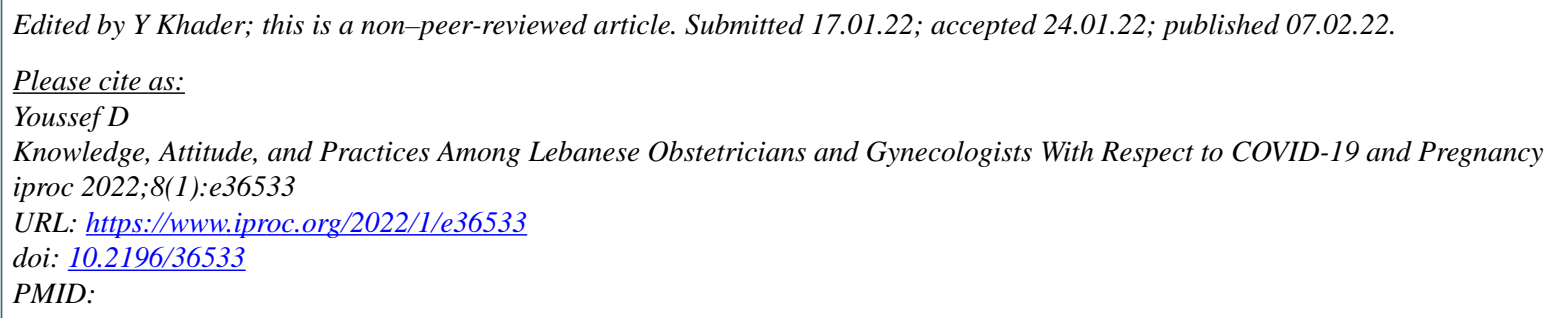


CDalal Youssef. Originally published in Iproceedings (https://www.iproc.org), 07.02.2022. This is an open-access article distributed under the terms of the Creative Commons Attribution License (https://creativecommons.org/licenses/by/4.0/), which permits unrestricted use, distribution, and reproduction in any medium, provided the original work, first published in Iproceedings, is properly cited. The complete bibliographic information, a link to the original publication on https://www.iproc.org/, as well as this copyright and license information must be included. 